Revista Científica do Curso de Pós-graduação Stricto Sensu em Turismo e Hotelaria da Universidade do Vale do Itajaí - UNIVALI Volume 15 - n. 2 - Mai./Ago. 2013

\author{
Reitor \\ Prof. Dr. Mário César dos Santos \\ Vice-Reitora \\ Profa. Dra. Amândia Maria de Borba \\ Procurador Geral \\ Vilson Sandrini Filho, MSc. \\ Secretário Executivo \\ Prof. Mércio Jacobsen, MSc. \\ Pró-Reitora de Ensino \\ Profa. Dra. Cássia Ferri \\ Pró-Reitor de Pesquisa, Pós-Graduação, \\ Extensão e Cultura \\ Prof. Dr. Valdir Cechinel Filho \\ Departamento de Pós-Graduação \\ Prof. Dr. Rogério Corrêa \\ Gerente de Campus em Balneário Camboriú \\ Cleunice Aparecida Trai \\ Editores da Revista \\ Profa. Dra. Josildete Pereira de Oliveira \\ Prof. Dr. Luiz Carlos da Silva Flores \\ Apoio \\ Carlos Eduardo de Almeida Ramoa \\ Letícia Indart Franzen \\ Coordenador da Editora \\ Prof. Dr. Rogério Corrêa \\ Secretária \\ Francine Lucatelli \\ Assistente de Marketing \\ Ricardo Luiz Aoki \\ Revisão \\ Profa. Ana Cláudia Reiser de Melo, MSc. \\ Diagramação \\ Francine Lucatelli \\ Livraria Universitária \\ Bárbara Christine Hass \\ Danielle Gamba da Silva Costa \\ Tradução \\ Profa. Fiona Oliver Robson, MSc. \\ Profa. Cynthia C. E. Philipps, MSc. \\ Prof. Daniel Paglialunga, MSc. (NELLE) - Espanhol \\ Indexadores e Diretórios \\ Latindex - 9115 \\ ULRICH'S Periodicals Directory \\ CIRET - Centre International de Recherches et \\ d'Etudes Touristiques \\ Sistema Integrado de Bibliotecas da Univali - \\ SIBIUN EBSCO
}

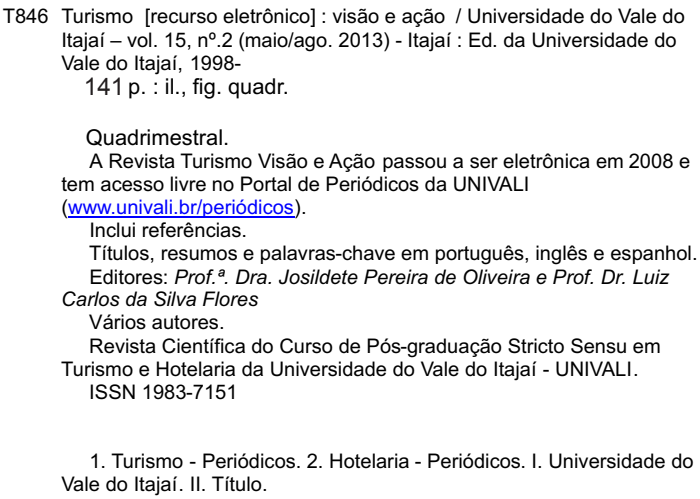

T846 Turismo [recurso eletrônico] : visão e ação / Universidade do Vale do Itajaí - vol. $15, n^{\circ} .2$ (maio/ago. 2013) - Itajaí : Ed. da Universidade do Vale do Itajaí, 1998

141 p. : il., fig. quadr.

Quadrimestral.

A Revista Turismo Visão e Ação passou a ser eletrônica em 2008 e tem acesso livre no Portal de Periódicos da UNIVAL

(www.univali.br/periódicos)

Inclui referências.

Títulos, resumos e palavras-chave em português, inglês e espanhol.

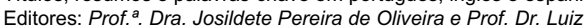
Carlos da Silva Flores

Vários autores.

Revista Cientifica do Curso de Pós-graduação Stricto Sensu em

Turismo e Hotelaria da Universidade do Vale do Itajaí - UNIVALI. ISSN 1983-7151

1. Turismo - Periódicos. 2. Hotelaria - Periódicos. I. Universidade do Vale do Itajaí. II. Título.

Conselho da Política Editorial

Carlos Alberto Tomelin (Diretor do Centro)

Francisco Antonio dos Anjos (Coordenador)

Josildete Pereira de Oliveira (Editora)

Luciano Torres Tricárico (Repres. LP I)

Luiz Carlos da Silva Flores (Repres. LP II)

Paulo dos Santos Pires (Editor)

\section{Corpo Editorial Científico}

Alexandre Panosso Netto (USP)

Anete Alberton (UNIVALI)

Carlos Manuel Martins da Costa (UALG) Cássia Ferri (UNIVALI)

Cléria Botêlho da Costa (UnB) Colin Michael Hall

(Universidade de Canterbury - Nova Zelândia) Dimitrius Buhalis

(Universidade de Bournemouth - Inglaterra)

Dóris Van de Meene Ruschmann (UNIVALI)

Eduardo Abdo Yázigi (USP)

Elizabeth Kioko Wada (UAM)

Gino Giacominni Filho (USCS)

Jaume Guia (Girona - Espanha)

José Manoel Gândara (UFPR)

José Fernando Vera Rebollo

(Universidade de Alicante - Espanha)

José Manuel Gonçalves Gândara (UFPR) Júlio Mendes (UALG)

Luzia Neide Menezes Teixeira Coriolano (UECE) Marcelino de Souza (UFRGS)

Margarita Nilda Barretto Angeli (FURB) Mario Carlos Beni

(Presidente CNT - Conselho Nacional de Turismo) Marta de Azevedo Irving (UFRJ) Mirian Rejowski (UAM) Neila Cabral (IFPA)

Raquel Maria Fontes do Amaral Pereira (UNIVALI) Regina Schluter (UQ - Argentina)

Roberto dos Santos Bartholo Júnior (UFRJ) Rosana Mara Mazaro (UFRN)

Sara Joana Gadotti dos Anjos (UNIVALI)

Suzana de Araújo Gastal (UCS) Thomas G. Baum

(Sthathclyde- Glasgow - Escócia)

Yolanda Flores e Silva (UNIVALI)

Submissões de trabalhos / Work submission http://www.univali.br/revistaturismo

E-mail: revistaturismo@univali.br

Endereço Editora

Rua Uruguai, 458 Caixa Postal 360

Cep: 88302 - 202 Itajaí - SC Fone: (47) 3341-7645

www.univali.br/editora - www.univali.br/periodicos e-mail: editora@univali.br - Twitter: @ed_univali 
Editorial

\section{ARTIGOS}

O ENSINO SUPERIOR DA GASTRONOMIA NO BRASIL: ANÁLISE DA REGULAMENTAÇÃO, DA DISTRIBUIÇÃO E DO PERFIL GERAL DE FORMAÇÃO (2010-2012)

Higher education in gastronomy in Brazil: an analysis of its regulation, distribution and general educational profile (2010-2012)

La enseñanza superior de la gastronomía en Brasil: análisis de la reglamentación, de la distribución y del perfil general de formación (2010-2012)

.166

Rebeca Elster Rubim

Mirian Rejowski

VOLUNTURISMO: UMA ABORDAGEM CONCEITUAL

Volunturismo: a conceptual approach

Volunturismo: un abordaje conceptual

Thaís Cristine Mendes

Kerlei Eniele Sonaglio

O turismo e a sua atuação na expansão do espaço urbano: o CASO Porto de Galinhas - IPOJUCA -PE

Tourism and its role in the expansion of the urban space: the case of Porto de Galinhas - Ipojuca-PE

El turismo y su papel en la expansión del espacio urbano: el caso Porto de Galinhas - Ipojuca -PE

Danielle Mesquita

Gilca Xavier

GESTÃO AMBIENTAL COMO ESTRATÉGIA DE MARKETING EM UMA EMPRESA DE TURISMO RURAL

Tourism management as a marketing strategy in a rural tourism company

La gestión ambiental como estrategia de marketing en una empresa de turismo rural 
PRESERVAÇÃO PATRIMONIAL, TURISMO CULTURAL E TRANSFORMAÇÃO DA BASE ECONÔMICA LOCAL: PIONEIRISMOS, PARADOXOS E RETROCESSOS EM WigAN, INGLATERRA

Heritage preservation, cultural tourism and transformation of the local economic base: pioneering, paradoxes and steps backwards in Wigan, England

Preservación patrimonial, turismo cultural y transformación de la base económica local: pioneirismos, paradojas y retrocesos en Wigan, Inglaterra

André Fontan Köhler

Políticas de GESTÃo de PESSOAS DESTINAdAS AOS PROFISSIONAIS COM DEFICIÊNCIA: UM ESTUDO EM UMA ORGANIZAÇÃO HOTELEIRA DA CIDADE DO Rio DE JANEIRO

Personnel management policies for people with disabilities: a study in a hotel organization in the city of Rio de Janeiro

Políticas de gestión de personas destinadas a los profesionales con discapacidad: un estudio en una organización hotelera de la ciudad de Río de Janeiro

Marta Pereira Forte Araújo

Carolina Lescura de Carvalho Castro

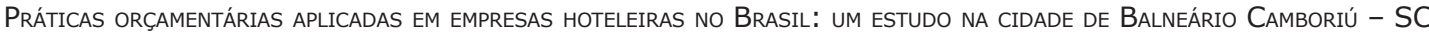

Budget practices applied to hotel companies in Brazil: a study in the town of Balneário Camboriú

Las prácticas presupuestarias aplicadas en empresas hoteleras en Brasil: un estudio en la ciudad de Balneário Camboriú

Monitoramento de impactos ambientais na trilha do Salto Ventoso, Farroupilha - RS

Monitoring environmental impacts on the Salto Ventoso trail, Farroupilha - RS

Monitorización de impactos ambientales en el sendero de Salto Ventoso, Farroupilha - RS 